gr-qc/9407006

NIKHEF-H/94-03

July 1994

\title{
EXTENDED GEOMETRY OF BLACK HOLES
}

\author{
K. Peeters, C. Schweigert and \\ J.W. van Holten \\ NIKHEF/FOM \\ P.O. Box 41882 \\ 1009 DB Amsterdam NL
}

\begin{abstract}
.
We reconsider space-time singularities in classical Einsteinian general relativity: with the help of several new co-ordinate systems we show that the Schwarzschild solution can be extended beyond the curvature singularity at $r=0$. The extension appears as an infinite covering of standard Kruskal space-time. While the twodimensional reduction of this infinite sequence of Kruskal-Szekeres domains obtained by suppressing the angular degrees of freedom is still a topological manifold - albeit one for which the metric structure is singular on one-dimensional submanifolds we obtain for the full four-dimensional geometry the more general structure of a stratified variety.
\end{abstract}

In this letter we present some remarks on the structure of space-time near singularities in classical, pure Einsteinian general relativity, i.e. the classical field theory for the metric structure defined by the Einstein equations. This theory in itself does certainly not describe all aspects of gravity, especially not at small length scales where quantum corrections should be taken into account. However, for any quantum theory of gravity a good understanding of the classical theory is helpful; in addition, the theory has found important applications both in physics and in mathematics.

In mathematical relativity space-time is usually assumed (compare e.g. [1]) to be a smooth, connected, inextendable Hausdorff manifold $M$ with a smooth Lorentz metric. (In fact, for geodesics to be uniquely defined, it is only necessary to assume that the metric is $C^{2-}$, i.e. differentiable with locally Lipschitz continuous derivatives.) One definition of inextendability is to require that $M$ cannot be embedded isometrically in a larger Lorentz manifold of the same dimension. There is also the stronger notion of local inextendability [1]: $M$ is called locally 
inextendable if there is no open set $\mathcal{U}$ in $M$ with non-compact closure that has an extension in which the closure of the image of $\mathcal{U}$ is compact.

Inextendability is an important requirement coming from physics: an essential aspect of general relativity is that the geometry of space-time is subject to dynamics. But then it is compulsory to consider an inextendable space-time manifold: taking an extendable space-time amounts to stopping dynamics at an arbitrary point. Such arbitrariness affects the validity of any global result.

The geometry of space-time is usually studied by means of geodesics. From the point of view of physics this involves the idealization of 'test particles' which certainly is debatable. From a mathematical point of view geodesics are a rather natural tool since they are known to encode most of the geometric information about $M$. Therefore, in this letter, completeness of (time-like) geodesics will be taken as the criterion for inextendability.

The notion of inextendability depends, of course, strongly on the other requirements one imposes on $M$ : weakening one of them may imply that a manifold that is inextendable in the original axiomatic setting becomes extendable in the new one. Below we present some arguments that requiring $M$ to be a smooth manifold with a smooth metric is too restrictive and that one has to include singularities in the description of space-time. This in turn will allow for the extension of Schwarzschild space-time we are going to present.

The main mathematical observation behind our results is the simple fact that the solution of a differential equation - in our case the geodesic equation - can be regular even if the coefficients of this equation, given by the metric and its derivatives, have singular points.

Proposals to extend the Schwarzschild solution beyond the curvature singularity at $r=0$ have also been put forward by other authors [2, 3]. In fact there is a small and - in our opinion undeservedly - little-known tradition in this field; we will therefore also rederive with new tools and in a slightly different perspective some results that are in principle not quite new, but not so well known. Given the renewed interest in extensions of back hole solutions motivated by string theory and taking into account "the resistance to any change in the rules" [3] we present them in this letter as well.

Compared to previous work, our treatment is simpler both from a conceptual and from a computational point of view. While in [3] similar results have been obtained by embedding Schwarzschild space-time in a cosmical dust solution, our investigations rely on the introduction of several co-ordinate systems for the Schwarzschild solution which to our knowledge have not been presented so far. As simple co-ordinate systems have frequently triggered progress in general relativity, this might be an independent point of interest of our results. We will state carefully the rules for the extension of the geodesics; in particular we will point out a subtle difference between the two-dimensional reduction of the geometry obtained by suppressing the angular part and the full four-dimensional geometry. This difference implies that in contrast to the two-dimensional reduction where the extension is still a topological manifold - albeit one for which the metric structure is divergent at infinitely many isolated one-dimensional submanifolds - it is necessary in the full four-dimensional geometry to replace the structure of a manifold by the closely related structure of a stratified variety.

In the rest of this letter we first introduce new local co-ordinates for Schwarzschild spacetime. In these co-ordinates the geometry near the singularity is explored first by use of radial 
geodesics; in a second step also non-radial geodesics are considered. We conclude with a description of the global features of this extension of the Schwarzschild solution.

The Schwarzschild metric is the locally unique solution of the Einstein equations in empty space [4, 5] describing a spherically symmetric gravitational field, static outside the horizon $r_{H}=2 M$. In terms of the original $(r, t)$ co-ordinates the solution is singular at the horizon, but other co-ordinate systems are known extending the solution smoothly across the horizon all the way up to the curvature singularity at $r=0$. The most complete description of this kind is the one by Kruskal [6] and Szekeres [7]. This space-time is not geodesically complete; however it has the important property that the only obstruction to geodesic completeness are the curvature singularities: all geodesics can be extended indefinitely unless they reach the curvature singularity.

The line element characterising the Kruskal-Szekeres solution takes the form

$$
\frac{\mathrm{d} s^{2}}{4 M^{2}}=f(u, v)\left(-\mathrm{d} v^{2}+\mathrm{d} u^{2}\right)+g(u, v) \mathrm{d} \Omega^{2},
$$

where $f(u, v)$ and $g(u, v)$ are functions of $u^{2}-v^{2}$ only, and the connection with the standard Schwarzschild co-ordinate is

$$
u^{2}-v^{2}=\left(\frac{r}{2 M}-1\right) \mathrm{e}^{r / 2 M}, \quad \frac{v}{u}= \begin{cases}\tanh \frac{t}{4 M}, & \text { if }|v|<|u| \\ \operatorname{coth} \frac{t}{4 M}, & \text { if }|v|>|u|\end{cases}
$$

The functions $f$ and $g$ are then implicitly defined by

$$
f(u, v)=\frac{8 M}{r} \mathrm{e}^{-r / 2 M}, \quad g(u, v)=\frac{r^{2}}{4 M^{2}} .
$$

The space-time domain covered by this solution of the Einstein equations consists of two physically distinct exterior regions, extending from $r=\infty$ to the horizon and usually denoted as regions I and III, and two interior regions bounded by the horizon and either the future or the past singularity, known as regions II and IV, respectively [8].

A new co-ordinate system for the Schwarzschild solution is given for the two regions I, III outside the black hole horizon $(r>2 M)$ by the line element

$$
\mathrm{d} s^{2}=-\tanh ^{2} \frac{\rho}{2} \mathrm{~d} t^{2}+4 M^{2} \cosh ^{4} \frac{\rho}{2}\left(\mathrm{~d} \rho^{2}+\mathrm{d} \Omega^{2}\right) .
$$

It is easily checked that this is a solution of the matter-free Einstein equations with $r>2 M$ by performing the transformation to standard $(r, t)$ co-ordinates:

$$
\frac{r}{2 M}=\cosh ^{2} \frac{\rho}{2} \text {. }
$$

The explicit connection of $\rho$ with the Kruskal-Szekeres co-ordinates is

$$
u=\mathrm{e}^{1 / 2 \cosh ^{2} \frac{\rho}{2}} \sinh \frac{\rho}{2} \cosh \frac{t}{4 M}, \quad v=\mathrm{e}^{1 / 2 \cosh ^{2} \frac{\rho}{2}} \sinh \frac{\rho}{2} \sinh \frac{t}{4 M} .
$$


In the domain $\rho=\rho_{+}>0$ this corresponds to $u>0$, and one obtains a cover of region I in the Kruskal-Szekeres diagram. Setting $\rho=\rho_{-}<0, \rho_{-}$covers the exterior region III, with $u<0$. The double covering of the exterior region $r>2 M$ in the Kruskal-Szekeres diagram is well-known to describe two physically distinct sheets of Schwarzschild space-time.

We observe that the co-ordinate system (丯 can be continued to the interior region of Schwarzschild space-time by a simple Wick rotation.

$$
\rho=\mp \text { i } \rho_{ \pm} \text {. }
$$

The two possible choices for the sign in the Wick rotation correspond to two possible ways of patching regions outside the horizon with $r>2 M$ to the interior of a black hole, yielding region I and III respectively. The line element inside the black-hole horizon $(r<2 M)$ is then given by

$$
\mathrm{d} s^{2}=\tan ^{2} \frac{\rho}{2} \mathrm{~d} t^{2}+4 M^{2} \cos ^{4} \frac{\rho}{2}\left(-\mathrm{d} \rho^{2}+\mathrm{d} \Omega^{2}\right)
$$

for $0 \leq \rho \leq 2 \pi$. This shows that after the Wick rotation $\rho$ becomes a time-like and $t$ a space-like co-ordinate, analogously to ordinary Schwarzschild co-ordinates. Comparing with equation (5), the connection with the usual $(r, t)$ co-ordinates is made by the transformation

$$
\cos ^{2} \frac{\rho}{2}=\frac{r}{2 M}
$$

Clearly, this provides a double-valued parametrization of the interior region containing the singularity $r=0$, or $\rho=\pi$ : every value $r>0$ corresponds to two distinct values of $\rho$ in the domain $[0,2 \pi]$. This double-valuedness holds both near the past and the future horizon, as follows from the transformation to Kruskal-Szekeres co-ordinates

$$
u=\mathrm{e}^{1 / 2 \cos ^{2} \frac{\rho}{2}} \sin \frac{\rho}{2} \sinh \frac{t}{4 M}, \quad v=\mathrm{e}^{1 / 2 \cos ^{2} \frac{\rho}{2}} \sin \frac{\rho}{2} \cosh \frac{t}{4 M} .
$$

In order to interpret the double covering of the region inside the horizon by our solution, we are going to investigate time-like geodesics. For simplicity let us start with incoming radial geodesics; they are solutions of the equation

$$
\frac{\mathrm{d} \rho}{\mathrm{d} t}=\frac{-\sin \frac{\rho}{2}}{2 M \cos ^{4} \frac{\rho}{2}},
$$

from which we deduce the following expression for the proper time measured by an infalling test particle

$$
\mathrm{d} \tau=2 M \cos ^{2} \frac{\rho}{2} \sin \frac{\rho}{2} \mathrm{~d} \rho,
$$

with $\tau$ increasing as $\rho$ increases from 0 at the horizon to $\pi$ at the singularity.

Moving now on to values $\rho>\pi$ and increasing $\tau$ according to eq.(12) we see that the particle moves away from the singularity as its proper time increases, and the region of $(u, v)$ space we are in contains a past singularity; hence it is physically distinct from the region before the encounter with the infinite curvature singularity. The double-valuedness thus connects the interior of the original Schwarzschild space-time at the curvature singularity with a new region 
that can be interpreted as the inner region of the white hole of a new Schwarzschild space-time. We will discuss the global features of such an extended space-time in more detail below.

We emphasize that we are not extending the $r$ co-ordinate to negative values, nor the Kruskal-Szekeres co-ordinates to $v^{2}-u^{2}>1$. Rather, we patch a new, physically distinct, region of positive $r$-values to space-time, which can be reached only by passing the curvature singularity.

In the sequel, we show that this extension can be done without running into physical paradoxes. It follows from eq.(12) that two points on a geodesic separated by the singularity are only a finite interval of proper time apart [2]:

$$
\int_{\rho=\pi-\varepsilon}^{\rho=\pi+\varepsilon} \mathrm{d} \tau=\frac{8 M}{3} \cos ^{3}\left(\frac{\pi-\varepsilon}{2}\right) \approx \frac{M}{3} \varepsilon^{3} .
$$

We also observe that the geodesic is smooth everywhere in the sense that the velocity as measured in the Kruskal-Szekeres co-ordinates is finite and continuous, even when the proper velocity and acceleration become momentarily infinite, and that the light-cone structure is preserved upon crossing the singularity.

In support of these statements, we consider a system of test particles falling in radially from rest at any point $0<r_{0}<\infty$. To construct the corresponding geodesics it is most convenient to introduce a collection of co-ordinate systems $(\kappa, t, \Omega)$ parametrized by a variable $\kappa_{H}$ of the form

$$
\mathrm{d} s^{2}=-\frac{\kappa^{2}-\kappa_{H}^{2}}{\left(1+\kappa_{H}^{2}\right) \kappa^{2}} \mathrm{~d} t^{2}+4 M^{2} \frac{\left(1+\kappa_{H}^{2}\right)^{2} \kappa^{4}}{\kappa_{H}^{4}\left(1+\kappa^{2}\right)^{2}}\left[\frac{1+\kappa_{H}^{2}}{\left(1+\kappa^{2}\right)^{2}} \frac{4 \mathrm{~d} \kappa^{2}}{\kappa^{2}-\kappa_{H}^{2}}+\mathrm{d} \Omega^{2}\right] .
$$

These co-ordinate systems describe Schwarzschild space-time for $r<r_{0}$ via the transformation

$$
\frac{r}{2 M}=\frac{r_{0}}{2 M} \frac{\kappa^{2}}{1+\kappa^{2}},
$$

with $r_{0}$ related to the parameter $\kappa_{H}$, the value of $\kappa$ at the horizon, by

$$
\frac{r_{0}}{2 M}=\frac{1+\kappa_{H}^{2}}{\kappa_{H}^{2}} .
$$

Just like the $(\rho, t)$ co-ordinate system, the $(\kappa, t)$ co-ordinate systems represent a double cover of the domain $0<r<r_{0}$, and in fact extend into the new region of space-time beyond the singularity. In terms of $\kappa$ the equation for radial geodesics becomes

$$
\frac{1}{4 M} \frac{\mathrm{d} t}{\mathrm{~d} \kappa}=\frac{\left(1+\kappa_{H}^{2}\right)^{2}}{\kappa_{H}^{3}} \frac{\kappa^{4}}{\left(1+\kappa^{2}\right)^{2}\left(\kappa_{H}^{2}-\kappa^{2}\right)} .
$$

For $\kappa<\kappa_{H}$ this has the solution

$$
\frac{t-t_{0}}{4 M}=\operatorname{arctanh} \frac{\kappa}{\kappa_{H}}-\left(\frac{1+3 \kappa_{H}^{2}}{2 \kappa_{H}^{3}}\right) \arctan \kappa+\left(\frac{1+\kappa_{H}^{2}}{2 \kappa_{H}^{3}}\right) \frac{\kappa}{1+\kappa^{2}} .
$$


It follows that the horizon at $t \rightarrow \infty$ is crossed at $\kappa=\kappa_{H}$, whilst the geodesic reaches the singularity $\kappa=0$ at $t=t_{0}$. Inside the horizon the velocity of the test particle in the KruskalSzekeres co-ordinate system is found to be

$$
\frac{\mathrm{d} u}{\mathrm{~d} v}=\tanh \left[\frac{t_{0}}{4 M}-\left(\frac{1+3 \kappa_{H}^{2}}{2 \kappa_{H}^{3}}\right) \arctan \kappa+\left(\frac{1+\kappa_{H}^{2}}{2 \kappa_{H}^{3}}\right) \frac{\kappa}{1+\kappa^{2}}\right] .
$$

Therefore $|\mathrm{d} u / \mathrm{d} v|<1$ at all times, both for $\kappa>0$ (before reaching the singularity) and for $\kappa<0$ (after traversing the singularity). On the other hand, null geodesics (light rays) correspond to straight lines

$$
\frac{\mathrm{d} u}{\mathrm{~d} v}= \pm 1
$$

It follows that time-like geodesics remain time-like also in the region of space-time corresponding to $\kappa<0$. Equation (19) proves the above assertion regarding the finiteness and continuity of the velocity in the $(u, v)$ co-ordinate system for all values of $\kappa$.

So far we have only considered radial geodesics; our results show that the two-dimensional geometry obtained by suppressing the angular degrees of freedom looks as follows: we obtain an infinite sequence of (two-dimensional reductions of) Kruskal-Szekeres space-times as depicted in figure 1 which forms a smooth topological manifold. However, the metric structure of this manifolds diverges along infinitely many one-dimensional submanifolds, marked by bold lines in figure 1. It can be shown that also the black hole solution for two-dimensional dilaton gravity can be extended similarly [9].

In a second step we are going to explore the extension of the angular co-ordinates $\varphi$ and $\theta$; let us therefore now have a look at non-radial geodesics. It is crucial to remark that angular momentum is a conserved quantity in our problem. Using this, we can choose $\theta=\frac{\pi}{2}=$ const on both sheets. Chosing this constant to be the same amounts to imposing the conservation law for angular momentum across the singularity.

In addition, we have the conserved quantity

$$
L=\cos ^{4} \frac{\rho}{2} \frac{\mathrm{d} \varphi}{\mathrm{d} \tau} .
$$

which allows to derive for geodesics with non-vanishing angular momentum a relation between $\rho$ and $\varphi$ that can be integrated across the singularity.

For geodesics in the equatorial plane entering from infinity we obtain

$$
\left(\frac{\mathrm{d} \rho / \mathrm{d} \tau}{\mathrm{d} \varphi / \mathrm{d} \tau}\right)^{2}=1+\frac{4 M^{2} \cos ^{4} \frac{\rho}{2}}{L^{2} \sin ^{2} \frac{\rho}{2}}
$$

which is finite in a neighbourhood of the singularity and becomes 1 in the limit $\rho \rightarrow \pi$. This implies the existence of a non-singular relation $\rho=\rho(\varphi)$, so that $\varphi$ can be integrated to a smooth function of proper time. For geodesics entering from a finite distance, an analogous argument goes through as well. However, the equations become more complicated and we refrain from presenting them in detail. 


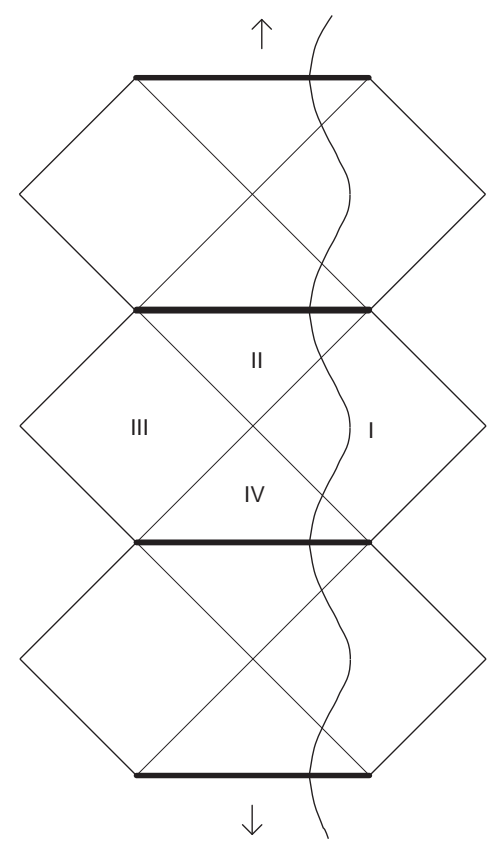

Figure 1: Schematic representation of the global structure of the extension of Schwarzschild space-time: an infinite sequence of Kruskal-Szekeres domains which are connected along lines of infinite curvature. The line depicts a radial geodesic crossing the singularities as described in the text.

The singularity can thus be interpreted as a single point on time-like geodesics at which the proper acceleration becomes momentarily infinite, as in the case of a test charge moving through the Coulomb singularity of a fixed charge of opposite sign. Just as in the electric analogue, a conservation law, the conservation of energy-momentum on geodesics, guarantees that in the absence of a hard (delta-function) core in the potential the particle continues its worldline after moving through the singular point, with finite velocity.

From this analogy, one might speculate that some of these features might survive in a quantum theory where it is well-known that singularities like the Coulomb singularity do not exclude the existence of well-behaved, normalizable particle wave-functions. Further support for this conjecture comes from recent work on two-dimensional black-hole models emerging from string theory, which are described by gauged Wess-Zumino-Witten sigma models [10, 11]. These theories also exhibit a pattern of geodesic continuation of space-time to new regions. Our results show that already in purely classical gravity such space-time extensions occur, even in the presence of curvature singularities.

Observing that in the new region of space-time there is a past singularity, it follows that eventually the geodesics reach another event-horizon, where the solution (8) can again be 
continued to an exterior region with a metric structure of type (4), but this time the Wick rotation is made around the point $2 \pi$, rather than $\rho=0$. Patching together these solutions in a new Kruskal-Szekeres co-ordinate system, one encounters a new future singularity, and the construction described here can be repeated indefinitely, both in the forward and backward directions of proper time. This is particularly clear if one follows one of the geodesics starting from rest at a finite distance $r_{0}$, as described by eq.(18); passing through the singularity at $r=0$ it reaches again a maximal distance $r_{0}$ in the new sheet of space-time, then falls back in until it reaches the next singularity, etc. In order to avoid problems with causality due to closed time-like loops we cannot identify the new regions with any earlier ones, a procedure which would result in a covering of space-time by a finite number of Kruskal-Szekeres space-times. We are thus forced to consider an infinite covering.

We are now in a position to describe the global geometric features of this extension of Schwarzschild space-time: it consists of an infinite sequence of Kruskal-Szekeres domains, connected along lines of infinite curvature. This geometrical object is not a manifold any more, but rather a stratified variety; by definition, a stratified variety is a connected topological space which can be represented as the disjoint sum of manifolds which can have different dimensions. The manifolds that make up this variety are referred to as strata. A simple example of a stratified variety is a double cone: it has a two-dimensional stratum consisting of two parts that are isomorphic separately to an infinitely long cylinder, and a zero-dimensional stratum, the tip of the cone.

In our case there are two strata: a four-dimensional one consisting of a disjoint sum of countably many Kruskal-Szekeres space-times, and one-dimensional stratum which consists of the singular lines (marked by the bold lines in figure 11). In fact, there is a two-dimensional sphere of radius $r$ attached to any point of the Kruskal diagram which degenerates at the singularity $r=0$, the one-dimensional stratum. The strata are glued together such that spacetime as a whole is connected; as we have seen, any timelike geodesic can be continued naturally through the singular stratum. This again has an analogue for the cone (supplied with the flat metric on it): there are two types of geodesics on a cone, curves that could be described as slightly curved hyperbolae and straight lines through the tip. The latter can be extended smoothly to the second sheet of the cone. However, in contrast to Schwarzschild space-time, only these radial geodesics cross the singularity.

In the stratified variety we propose as the extension of Schwarzschild space-time all geodesics can be continued indefinitely in a unique way, including those that reach the curvature singularity; therefore the resulting space-time is geodesically complete and should be seen as the truly inextendable space-time underlying Schwarzschild geometry.

\section{References}

[1] S.W. Hawking and G.F.R. Ellis, The Large Scale Structure of Space Time Cambridge Univ. Press (Cambrigde, 1973)

[2] J.L. Synge, Proc. Roy. Irish Acad. 53 (1950) 83

[3] D. Lynden-Bell and J. Katz, Mon. Not. Roy. Astr. Soc. 247 (1990), 651 
[4] K. Schwarzschild, Sitz. Preuss. Akad. Wiss. Berlin, Kl. Math. Phys. Tech. (1916), 189

[5] J. Droste, PhD. Thesis, Leiden Univ. (1916)

[6] M.D. Kruskal, Phys. Rev. 119 (1960), 1743

[7] G. Szekeres, Publ. Mat. Debrecen 7 (1960), 285

[8] S. Chandrasekhar, The Mathematical Theory of Black Holes, Clarendon Press (Oxford, 1983); I. Novikov and V.P. Frolov, Physics of Black Holes, Kluwer Ac. Publ. (Dordrecht, 1989)

[9] K. Peeters, Thesis, University of Amsterdam, 1994

[10] E. Witten, Phys. Rev. D 44 (1991), 314

[11] R. Dijkgraaf, H. Verlinde and E. Verlinde, Nucl. Phys. B371 (1992), 269 